Article

\title{
Characterization of Responsive Hydrogel Nanoparticles upon Polyelectrolyte Complexation
}

\author{
Su-Kyoung Lee ${ }^{1}$, Gyuri Hwang ${ }^{2}$, Jihyun Woo ${ }^{2}$, Joseph Park ${ }^{2}$ and Jongseong Kim ${ }^{1,2, *}$ \\ 1 Yonsei-IBS Institute, Yonsei University, Seoul 03722, Korea; switfrik@gmail.com \\ 2 STEM Research Institute, Fairfax, VA 22031, USA; paulschung08@gmail.com (G.H.); \\ joo1123kr@gmail.com (J.W.); hydrom7@daum.net (J.P.) \\ * Correspondence: envokim72@yonsei.ac.kr; Tel.: +82-2-2123-7671
}

Academic Editor: Francoise Winnik

Received: 10 January 2017; Accepted: 13 February 2017; Published: 16 February 2017

\begin{abstract}
Characterization of responsive hydrogels and their interaction with other molecules have significantly expanded our understanding of the functional materials. We here report on the response of poly ( $\mathrm{N}$-isopropylacrylamide-co-acrylic acid) (pNIPAm-co-AAc) nanogels to the addition of the poly(allylamine hydrochloride) (PAH) in aqueous dispersions. We find that the hydrodynamic radius and stability of nanogels are dependent on the PAH/nanogel stoichiometry. If the nanogel solution is titrated with very small aliquots of $\mathrm{PAH}$, the nanogels decrease in radius until the equivalence point, followed by aggregation at suprastoichiometric PAH additions. Conversely, when titrated with large aliquots, the nanogel charge switches rapidly from anionic to cationic, and no aggregation is observed. This behavior correlates well with electrophoretic mobility measurements, which shows the nanogel charge transitioning from negative to positive upon PAH addition. The volume phase transition temperature (VPTT) of the nanogels is also measured to discover the effect of polyelectrolyte complexation on the deswelling thermodynamics. These data show that charge neutralization upon PAH addition decreases the VPTT of the nanogel at $\mathrm{pH}$ 6.5. However, if an excess amount of PAH is added to the nanogel solution, the VPTT shifts back to higher temperatures due to the formation of a net positive charge in the nanogel network.
\end{abstract}

Keywords: hydrogel; nanogel; polyelectrolyte; aggregation; deswelling; swelling

\section{Introduction}

Stimuli-responsive hydrogels have been investigated on various purposes due to the capability of hydrogels in response to external stimuli by physicochemical changes in the materials [1-9]. This responsivity is manifested in the swelling/deswelling of the polymer network as a function of temperature or $\mathrm{pH}$ [10-12]. In the swollen state, the polymer-solvent interactions are favorable, where in the specific case of poly( $N$-isopropylacrylamide) (pNIPAm), these interactions are favorable below the VPTT of $31{ }^{\circ} \mathrm{C}$, which is analogous to the coil-to-globule transitions seen in linear polymer systems as the lower critical solution temperature (LCST) is exceeded [13]. AAc copolymers are additionally $\mathrm{pH}$-responsive, where at $\mathrm{pH}$ values higher than $\mathrm{p} K_{\mathrm{a}}$ (4.25) [14] of AAc, the network tends to swell further due to Coulombic repulsion between the deprotonated acid groups, also resulting in an increase in the VPTT [15]. The favorable polymer-water interactions in pNIPAm can be disrupted by increasing the temperature of the system above the VPTT. Under these conditions, it is entropically favorable for the network to expel water and hydrophobically aggregate, resulting in a large-magnitude decrease in the network volume. The swelling/deswelling due to changes in $\mathrm{pH}$ is less abrupt, where the responsivity is due to Coulombic repulsion and an increase in the Donnan potential inside the network as the AAc groups become charged. It has also been reported that specifically engineered hydrogels with additional functionalities are responsive to stimuli such as $\mathrm{pH}$, ionic strength, photon flux, and 
biomolecular binding events [16-18]. These responsive hydrogels have been studied extensively for numerous applications, such as controlled drug release [19-22], tissue regeneration [5], surface patterning [23], microfluidic flow control [4], tunable optics [7,16,17,24], and molecular switches [25].

Polyelectrolyte complexation is an intriguing means useful for many applications by taking advantage of simple charge-charge interactions between polyelectrolytes, which includes not only the formation of physically cross-linked hydrogel particles [26,27] in drug delivery [28-30] but also fabrication of micro-capsules by building up polymeric multilayers on colloidal nanoparticles [31,32], followed by nanoparticle dissolution [33-35]. This phenomenon can also be exploited for the fabrication of thin films by the layer-by-layer (LbL) deposition approach. In addition, polyelectrolyte complex formation, known as complex coacervation, has been investigated to improve the applications [36], providing many interesting properties including low interfacial energy $[37,38]$ and viscoelastic response [39]. Polyelectrolyte complex systems are extensively utilized to improve biological applications in drug delivery [40,41], tissue engineering [42], and biological hybrid thin films [43,44]. Polyelectrolyte complexation in application of polymeric thin films composed of pNIPAm and AAc allows for determining the interaction/effects of various polyelectrolytes on the behavior (swelling and VPTT) of the thin film $[45,46]$. Polyelectrolytes can also be exploited for gene delivery where electrostatic interactions between the polyelectrolyte and DNA are used to form nanoparticles which can transfect cells $[47,48]$.

In this study, we explore the effect of added poly(allylamine hydrochloride) (PAH; $\mathrm{pKa}=9.67)$ [49] on the colloidal stability and thermoresponsivity of pNIPAm-co-AAc nanogels. The pNIPAm-co-AAc nanogels behave as polyanions and reservoirs for polycation adsorption, allowing for their complexation in aqueous dispersion.

\section{Materials and Methods}

\subsection{Materials}

All reagents were purchased from Sigma-Aldrich unless otherwise specified. The monomer $N$-isopropylacrylamide (NIPAm) was re-crystallized from hexanes (J.T. Baker ${ }^{\circledR}$, Center Valley, PA, USA) and dried under vacuum prior to use. Acrylic acid (AAc) was distilled under reduced pressure. The cross-linker $N, N^{\prime}$-methylene(bisacrylamide) (BIS), ammonium persulfate (APS), and sodium dodecyl sulfate (SDS) were used as received. Poly(allylamine hydrochloride) (PAH), $M_{\mathrm{w}} 70,000$, was used as received. All water used throughout this investigation was house distilled, deionized to a resistance of at least $18 \mathrm{M} \Omega$ (Barnstead Thermolyne E-Pure system, Thermo Fischer Scientific, Waltham, MA, USA), and then filtered through a $0.2 \mu \mathrm{m}$ filter for particulate removal.

\subsection{Nanogel Synthesis}

All nanogels used throughout this study were synthesized via aqueous free-radical precipitation polymerization, as previously described [50-52]. The syntheses were carried out by fixing the total monomer concentration constant at $70 \mathrm{mM}$ and the AAc mole percent constant at $10 \%$ while varying the BIS mole percent between $2 \%$ and $10 \%$ and adjusting the NIPAm concentration accordingly. Polymerization was performed in a three-neck, $250 \mathrm{~mL}$ round-bottom flask containing a magnetic stir bar. To this reactor, $100 \mathrm{~mL}$ of a filtered $(0.2 \mu \mathrm{m}$ filter, Pall Gelman Metricel, Port Washington, NY, USA), aqueous solution of NIPAm (697 or $622 \mathrm{mg}$ ), BIS (22 or $108 \mathrm{mg}$ ), and the surfactant SDS (28.8 mg, $1 \mathrm{mM}$ final concentration) was added. This solution was heated to $\sim 70{ }^{\circ} \mathrm{C}$ while degassing with $\mathrm{N}_{2}$ and stirring vigorously for $\sim 1 \mathrm{~h}$. After $1 \mathrm{~h}$, AAc was added to the flask in order to bring the total final monomer concentration up to $70 \mathrm{mM}$. Once the AAc was added, polymerization was immediately initiated by injection of $1.0 \mathrm{~mL}$ of an APS solution $(0.3 \mathrm{mmol})$. The solution turned turbid, indicating successful initiation. This solution was allowed to heat and stir for an additional $5 \mathrm{~h}$ while under a constant flow of $\mathrm{N}_{2}$ gas. Following synthesis, the particles were purified by dialysis against 
water for $\sim 2$ weeks with the water being changed twice per day, using 10,000 $M_{W}$ cut-off dialysis tubing (VWR).

\subsection{Nanogel Titration with PAH}

Titration of the nanogels used in this study was accomplished by adding aliquots of 0.0526 monoM (moles/L monomer) PAH solution ( $\mathrm{pH} 4.2$ with $10 \mathrm{mM} \mathrm{NaCl})$ to a dilute solution of nanogels $(10 \mu \mathrm{L}$ of purified nanogels diluted to $3.5 \mathrm{~mL}$ ), which is $2.0 \times 10^{-5} \mathrm{M} \mathrm{AAc}$, at $\mathrm{pH} 6.5$ (adjusted by $\mathrm{HCl}$ and $\mathrm{NaOH}$ with $10 \mathrm{mM} \mathrm{NaCl}$ ) contained in a plastic cuvette. Each nanogel solution was made immediately before each measurement. The radius and thermoresponsive behavior of the nanogels following titration was measured using Dynamic Light Scattering instrument (DLS, Protein Solutions Inc., Charlottesville, VA, USA).

\subsection{Dynamic Light Scattering (DSL) Analysis}

The use of DLS as a tool for determining mean particle size and particle size distributions has been described previously [53] and used for these nanogel systems in the past [50,52]. Briefly, the nanogel solution contained in a cuvette was inserted into the cuvette holder, which was equipped with a Peltier device for sample temperature control. Laser light $(783.9 \mathrm{~nm})$ was introduced to the sample via a single-mode optical fiber. Scattered light was collected by a fiber coupled avalanche photodiode detector at $90^{\circ}$. The scattered light can then be correlated to the translational diffusion coefficient of the nanogels, through the autocorrelation function, from which the hydrodynamic radius is calculated from the Stokes-Einstein equation. For equilibrium nanogel radius measurements, each sample was allowed to equilibrate at $25^{\circ} \mathrm{C}$ for $10 \mathrm{~min}$. The reported radius value was an average of 15 individual radius measurements using a $5 \mathrm{~s}$ integration time for each measurement. For VPTT measurements, the nanogels were first titrated with a given amount of PAH. The solution was then heated and the nanogel size determined every $2{ }^{\circ} \mathrm{C}$ by letting the sample equilibrate at each temperature for $10 \mathrm{~min}$. At each temperature, 5 consecutive runs were performed where each run was composed of 15 individual radius measurements using a $10 \mathrm{~s}$ integration time for each measurement.

\subsection{Electrophoretic Mobility Measurements}

Electrophoretic mobility was measured to follow nanogel charge upon PAH addition using a ZetaPlus instrument (Brookhaven Instruments Corporation, Holtsville, NY, USA). The samples were prepared in the same way they were prepared for DLS analysis and each reported electrophoretic mobility value is the mean value of 10 runs. The software provided by the instrument manufacturer was used to calculate the electrophoretic mobility.

\section{Results and Discussion}

The average nanogel hydrodynamic radius $\left(R_{h}\right)$ was determined by DLS as a function of PAH addition. As shown in Figure 1, for titration of 10\% AAc/2\% BIS nanogels, the radius decreases from $\sim 250$ to $\sim 100 \mathrm{~nm}$ upon the addition of $\mathrm{PAH}$, regardless of the PAH aliquot volume. In the case of 2.0 and $5.0 \mu \mathrm{L}$ PAH aliquot additions, the nanogel size then increases upon subsequent PAH addition to a radius of $\sim 250 \mathrm{~nm}$. This value is important to note because it is approximately the same as the particle radius at this $\mathrm{pH}$ in the absence of PAH. This swelling behavior is most likely due to the nanogel network structure becoming internally cross-linked by $\mathrm{AAc} / \mathrm{NH}_{2}$ interactions, followed by PAH saturation. The saturation of the particle with PAH should in turn produce an excess positive charge in the nanogel network, thereby resulting in Coulombic swelling. Another interesting feature in Figure 1 is the behavior seen upon adding $0.5 \mu \mathrm{L}$ PAH aliquots to the system. As the $[\mathrm{AAc}] /\left[\mathrm{NH}_{2}\right]$ ratio approaches 1 , the nanogel radius decreases, as seen in the case of the 2.0 and $5.0 \mu \mathrm{L}$ PAH aliquot additions. However, in the case of $0.5 \mu \mathrm{L}$ PAH aliquot addition, the nanogels tend to significantly increase in radius, indicating system aggregation rather than Coulombic swelling. Further evidence of aggregation comes from the dramatic increase in population breadth, as shown in 
the particle size histograms obtained from DLS (Figure S1). It is also interesting to note that there is no time dependency to the radius determination below the critical point, i.e., excess negative charge on the pNIPAm-co-AAc nanogel (Figures S2 and S3). As the point of neutrality is approached, the nanogels behave differently. The nanogel titrated with $0.5 \mu \mathrm{L}$ PAH aliquot shows aggregation in a time-dependent manner (Figure S2), whereas in the case of 5.0 $\mu \mathrm{L} \mathrm{PAH} \mathrm{aliquot} \mathrm{the} \mathrm{nanogel} \mathrm{remains} \mathrm{in}$ no time-dependent kinetics and aggregation (Figure S3). This is an indication that the nanogels are becoming instantly charge-reversed upon the addition of saturating amounts of PAH.

One way to explain this behavior is by considering the fact that, as the nanogels reach the neutralization point, the electrical repulsion between the nanogel particles is decreased and van der Waals attraction may dominate the nanogel dispersion in aqueous system. Thus, the energy barrier that prevents particle collisions may be decreased resulting in aggregation of the nanogels on the time scale of the experiment [54-56]. However, this mechanism is not the most likely source of aggregation, since water-swollen pNIPAm nanogels dispersed in water should have a low effective Hamaker constant and do not tend to aggregate at temperatures below the VPTT. Another possible explanation of this behavior relates to the homogeneity (or lack thereof) of absorption of PAH on the nanogels. It is most likely the case that the absorption of PAH is inhomogeneous as one slowly approaches the stoichiometric equivalence point, resulting in nanogels that have patchy-type charge distribution. Thus, when the nanogels collide they should have a higher tendency to aggregate at slightly suprastoichiometric $\mathrm{AAc} / \mathrm{NH}_{2}$ values $[55,57]$.

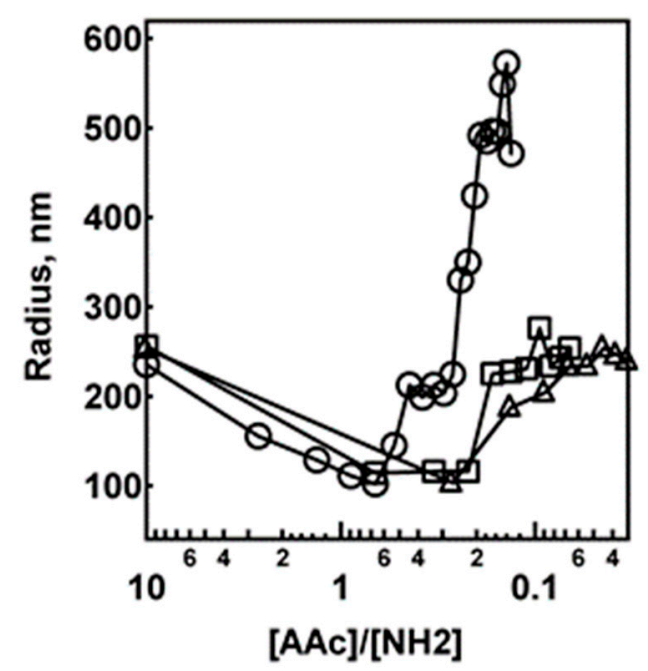

Figure 1. Nanogel titration plots for the titration of $2 \%$ BIS, $10 \%$ AAc nanogels upon the addition of $0.5(\bigcirc), 2.0(\square)$, and $5.0(\triangle)$ L PAH aliquots. The hydrodynamic radius of nanogels measured by DLS.

To determine if nanogel cross-link density has an effect on the interactions between PAH and AAc, titration experiments were conducted exactly as outlined above using nanogels containing $10 \%$ BIS and 10\% AAc. As shown in Figure 2, the same qualitative trends are obtained as were seen for the $2 \%$ BIS titrations. For the 2.0 and $5.0 \mu \mathrm{L} \mathrm{PAH} \mathrm{aliquot} \mathrm{additions,} \mathrm{the} \mathrm{nanogels} \mathrm{are} \mathrm{colloidally} \mathrm{stable} \mathrm{at}$ all stoichiometries, but in the case of the $0.5 \mu \mathrm{L}$ PAH aliquot addition, the nanogels again aggregate. One important difference that must be pointed out between the $10 \%$ and $2 \%$ BIS nanogel titrations is the magnitude of the deswelling upon PAH addition. In the $2 \%$ BIS case, the magnitude of collapse is much greater than that for the $10 \%$ BIS nanogels upon PAH addition. This is expected, as the $10 \%$ BIS nanogels have a greater network density than the $2 \%$ BIS nanogels, thus allowing for a smaller change in equilibrium swelling degree upon the addition of PAH. 


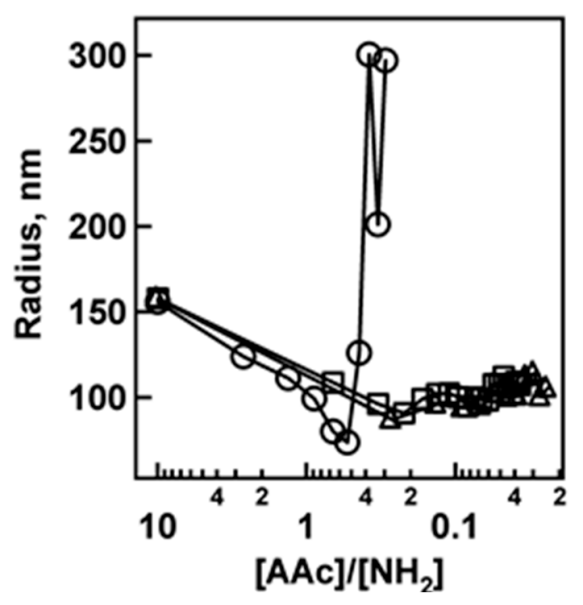

Figure 2. Nanogel titration plots for the titration of 10\% BIS, 10\% AAc nanogels upon the addition of $0.5(\bigcirc), 2.0(\square)$, and $5.0(\triangle) \mu \mathrm{L}$ PAH aliquots. The hydrodynamic radius of nanogels measured by DLS.

Electrophoretic mobility measurements were made for the pNIPAm-co-AAc nanogels containing $2 \%$ BIS upon the addition of 0.5 and $5.0 \mu \mathrm{L}$ PAH aliquots to verify the above claims of nanogel neutralization and charge reversal. To interpret the data it is important to keep in mind that uncharged (no AAc) pNIPAm nanogels have approximately zero electrophoretic mobility under the conditions that these experiments were conducted (Figure S4). The data in Figure 3 show that the nanogels initially display a negative electrophoretic mobility due to the deprotonated AAc groups on the pNIPAm-co-AAc nanogels. As $0.5 \mu \mathrm{L}$ PAH aliquots are titrated into the nanogel solution, the electrophoretic mobility slowly approaches zero. At $\sim 1: 1 \mathrm{AAc} / \mathrm{NH}_{2}$, the average electrophoretic mobility is approximately zero, as expected for a 1:1 acid/base stoichiometry. As more PAH is added to the system, the electrophoretic mobility then reverses to positive values due to the excess charge present in the nanogel. If, on the other hand, the electrophoretic mobility is followed as a function of $5.0 \mu \mathrm{L}$ PAH aliquot additions, the behavior is very different. This result is shown in Figure 4, where following the first $5.0 \mu \mathrm{L}$ PAH aliquot the average electrophoretic mobility reverses from a negative to a positive value. In light of these results, the data shown in Figures 1 and 2 are understandable in terms of effective surface charge arguments. If excess amounts of PAH are added to the system ( 2.0 and $5.0 \mu \mathrm{L}$ case) the nanogel rapidly reverses charge from negative to positive while never reaching an equilibrated point of instability (neutrality). In contrast, $0.5 \mu \mathrm{L}$ PAH aliquot additions cause the critical aggregation (neutralization) point to be reached slowly, whereupon the nanogels are able to aggregate due to the inhomogeneous charge distribution present on the nanogels.

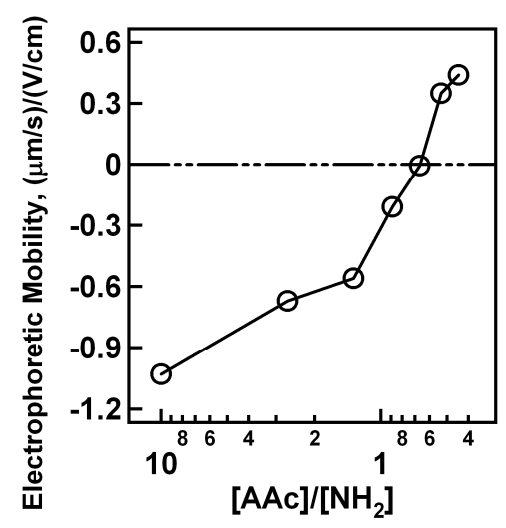

Figure 3. Electrophoretic mobility values as a function of $\mathrm{PAH}$ addition for $2 \%$ BIS and $10 \%$ AAc nanogels upon $0.5 \mu \mathrm{L}$ PAH aliquot additions. Note the $\mathrm{pH}$ of solutions adjusted at 6.5 with $10 \mathrm{mM} \mathrm{NaCl}$. 


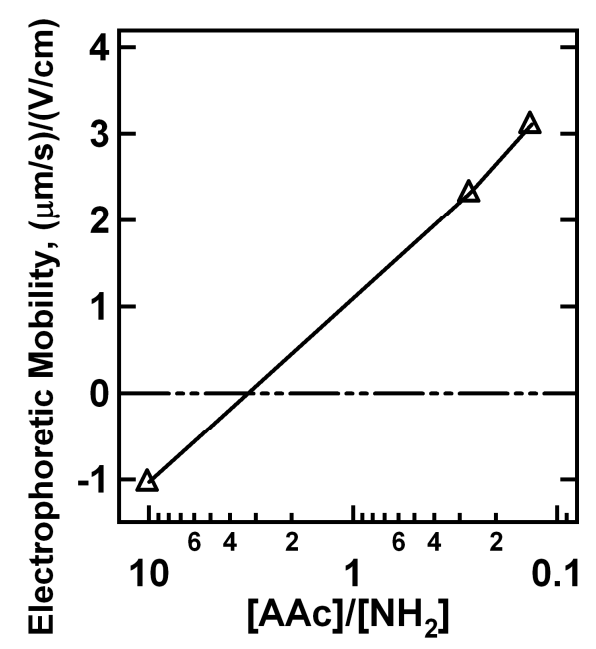

Figure 4. Electrophoretic mobility values as a function of $\mathrm{PAH}$ addition for $2 \% \mathrm{BIS}$ and $10 \%$ AAc nanogels upon $5.0 \mu \mathrm{L}$ PAH aliquot additions. Note the $\mathrm{pH}$ of solutions adjusted at 6.5 with $10 \mathrm{mM} \mathrm{NaCl}$.

To investigate the influence of PAH on the thermal deswelling of pNIPAm-AAc nanogels, we determined the particle size as a function of temperature at various nanogel/PAH stoichiometries (Figure 5). This figure shows a series of volume phase transition curves obtained by DLS for pNIPAm-co-AAc nanogels at $\mathrm{pH} 3.0$, where the particles are essentially electroneutral, and at $\mathrm{pH} 6.5$, where the particles are anionic due to AAc deprotonation. As shown in Figure 5, the pNIPAm-co-AAc nanogels at $\mathrm{pH} 3.0$ display a VPTT of $30{ }^{\circ} \mathrm{C}$, while at $\mathrm{pH} 6.5$ shifting the value to $\sim 54{ }^{\circ} \mathrm{C}[12,52,58]$. However, when pNIPAm-co-AAc nanogels at $\mathrm{pH} 6.5$ are titrated with $\mathrm{PAH}$ such that $\mathrm{AAc} / \mathrm{NH}_{2}$ is $~ 1: 1$, the nanogel VPTT reverts to $33^{\circ} \mathrm{C}$. This behavior suggests that the nanogel structure is becoming fully neutralized due to the AAc- $\mathrm{NH}_{2}$ interactions, where the $\mathrm{NH}_{2}$ groups are capable of neutralizing the deprotonated AAc sites inside the nanogel network. It is also interesting to note that the nanogel radius following PAH addition is somewhat smaller that the radius of the native nanogel at $\mathrm{pH} 3.0$ (125 nm vs. $150 \mathrm{~nm})$, suggesting that, in addition to charge neutrality, an effective increase in cross-link density arises from polyelectrolyte complexation. Figure 5 also shows the effect of the addition of excess PAH to the pNIPAm-co-AAc nanogels, where no VPTT is observed over this temperature range. This difference is most likely due to the excess PAH contributing to a net positive charge inside the nanogel structure, resulting in Coulombic repulsion inside the nanogel network, which then shifts the VPTT to a higher temperature. Our electrophoretic mobility results are in part suggestive of this, as it is clear that suprastoichiometric concentrations of PAH induce charge reversal. The DLS results additionally suggest that this charge reversal is not solely localized on the nanogel surface, as simple surface charging would not be expected to inhibit nanogel deswelling on the whole. For example, multiple papers on pNIPAm core/pNIPAm-co-AAc shell nanogels have shown that highly charged nanogel shells do not completely inhibit core deswelling. This observation indicates that charge reversal of the nanogels is accomplished with excess PAH addition. 


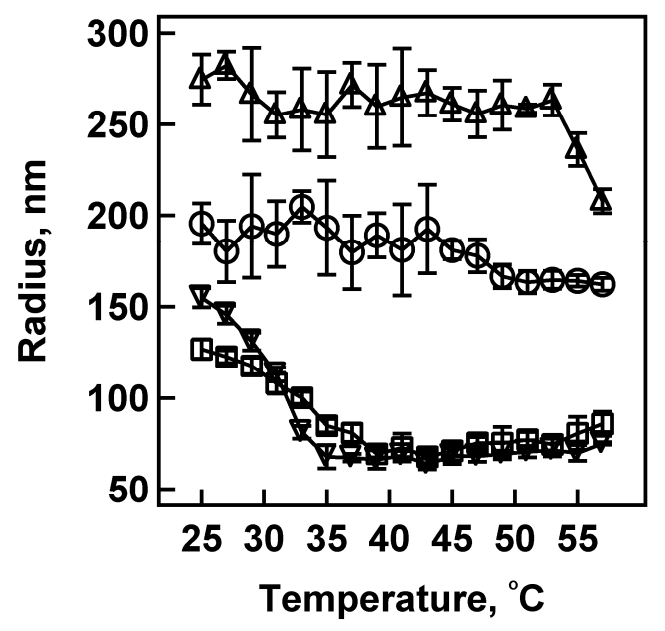

Figure 5. Volume phase transition curves for $2 \%$ BIS, 10\% AAc nanogels at $\mathrm{pH} 6.5(\triangle), \mathrm{pH} 3.0(\nabla)$, $\mathrm{pH} 6.5$ with $[\mathrm{AAc}] /\left[\mathrm{NH}_{2}\right]=0.9(\square)$, and $\mathrm{pH} 6.5$ with $[\mathrm{AAc}] /\left[\mathrm{NH}_{2}\right]=0.26(\bigcirc)$. The VPTT for nanogels at $\mathrm{pH} 6.5$ with $[\mathrm{AAc}] /\left[\mathrm{NH}_{2}\right]=0.9$ is very close to the VPTT observed for $\mathrm{pH}$ 3.0.

\section{Conclusions}

We have illustrated that loosely cross-linked pNIPAm-co-AAc nanogels in their deprotonated form bind with the linear polyelectrolyte PAH via ion pairing interactions. Light scattering and electrophoretic mobility measurements suggest that the hydrodynamic radius of pNIPAm-co-AAc nanogels is predominantly influenced not only by the cross-linking density of the hydrogels but also by the net charge of the gel network. This causes dramatic deswelling of the gels due to both charge compensation and cross-linking effects. It has also been demonstrated that the colloidal stability of the nanogels is impacted by the rate at which PAH is added to the dispersion. By approaching the point of stoichiometric equivalence slowly, a point of zwitterionic charge neutrality is obtained, resulting in particle aggregation. Conversely, large increases in PAH concentration overshoot this instability point and allow for charge reversal on the nanogel particle. Observing VPTT shifts as a function of PAH addition corroborates this behavior. When appropriate PAH is present to neutralize the charges inside the nanogels, the VPTT shifts to temperatures observed for neutralized nanogels at $\mathrm{pH}$ 3.0. If an excess amount of PAH is added into the nanogel solution, the VPTT shifts back to high temperatures illustrating the charge reversal phenomenon.

Supplementary Materials: The following are available online at www.mdpi.com/2073-4360/9/2/66/s1: Figure S1: Histograms for the 0.5 and $5.0 \mu \mathrm{L}$ PAH aliquot addition cases for $2 \%$ BIS cross-linked nanogels; Figure S2: Time dependent nanogel titration plot for the $2 \%$ BIS, 10\% AAc nanogels upon the addition of $0.5 \mu \mathrm{L}$ PAH aliquots at $0(\bigcirc), 210(\square), 420(\triangle)$, and $630(\nabla)$ s; Figure S3: Time dependent nanogel titration plot for the $2 \%$ BIS, 10\% AAc nanogels upon the addition of $5 \mu \mathrm{L}$ PAH aliquots at $0(\bigcirc), 210(\square), 420(\triangle)$, and $630(\nabla) \mathrm{s}$; Figure S4: Electrophoretic mobility values as a function of PAH addition for $2 \%$ BIS, 98\% NIPAm nanogels.

Acknowledgments: Jongseong Kim acknowledge support from National Research Foundation of Korea (NRF-2016R1D1A1B03933938).

Author Contributions: Jongseong Kim designed and performed the research; Jongseong Kim and Su-Kyoung Lee wrote the paper; Su-Kyoung Lee, Gyuri Hwang, Jihyun Woo, and Joseph Park performed the research.

Conflicts of Interest: The authors declare no conflict of interest.

\section{References}

1. Kwon, I.C.; Bae, Y.H.; Kim, S.W. Electrically erodible polymer gel for controlled release of drugs. Nature 1991, 354, 291-293. [CrossRef] [PubMed]

2. Aggeli, A.; Bell, M.; Boden, N.; Keen, J.N.; Knowles, P.F.; McLeish, T.C.; Pitkeathly, M.; Radford, S.E. Responsive gels formed by the spontaneous self-assembly of peptides into polymeric $\beta$-sheet tapes. Nature 1997, 386, 259-262. [CrossRef] [PubMed] 
3. Miyata, T.; Asami, N.; Uragami, T. A reversibly antigen-responsive hydrogel. Nature 1999, 399, $766-769$. [CrossRef] [PubMed]

4. $\quad$ Beebe, D.J.; Moore, J.S.; Bauer, J.M.; Yu, Q.; Liu, R.H.; Devadoss, C.; Jo, B.H. Functional hydrogel structures for autonomous flow control inside microfluidic channels. Nature 2000, 404, 588-590. [CrossRef] [PubMed]

5. Lutolf, M.P.; Lauer-Fields, J.L.; Schmoekel, H.G.; Metters, A.T.; Weber, F.E.; Fields, G.B.; Hubbell, J.A. Synthetic matrix metalloproteinase-sensitive hydrogels for the conduction of tissue regeneration: Engineering cell-invasion characteristics. Proc. Natl. Acad. Sci. USA 2003, 100, 5413-5418. [CrossRef] [PubMed]

6. Langer, R.; Tirrell, D.A. Designing materials for biology and medicine. Nature 2004, 428, 487-492. [CrossRef] [PubMed]

7. Holtz, J.H.; Asher, S.A. Polymerized colloidal crystal hydrogel films as intelligent chemical sensing materials. Nature 1997, 389, 829-832. [CrossRef]

8. Kiser, P.F.; Wilson, G.; Needham, D. A synthetic mimic of the secretory granule for drug delivery. Nature 1998, 394, 459-462. [PubMed]

9. Plunkett, K.N.; Berkowski, K.L.; Moore, J.S. Chymotrypsin responsive hydrogel: Application of a disulfide exchange protocol for the preparation of methacrylamide containing peptides. Biomacromolecules 2005, 6, 632-637. [CrossRef] [PubMed]

10. Tanaka, T.; Fillmore, D.J. Kinetics of swelling of gels. J. Chem. Phys. 1979, 70, 1214-1218. [CrossRef]

11. Dusek, K.; Patterson, K. Transition on swollen polymer networks induced by intramolecular condensation. J. Polym. Sci. 1968, 6, 1209-1216.

12. Pelton, R. Temperature-sensitive aqueous microgels. Adv. Colloid Interface Sci. 2000, 85, 1-33. [CrossRef]

13. Wang, X.; Qiu, X.; Wu, C. Comparison of the coil-to-globule and the globule-to-coil transitions of a single poly(N-isopropylacrylamide) homopolymer chain in water. Macromolecules 1998, 31, 2972-2976. [CrossRef]

14. Serpe, M.J.; Jones, C.D.; Lyon, L.A. Layer-by-layer deposition of thermoresponsive microgel thin films. Langmuir 2003, 19, 8759-8764. [CrossRef]

15. Yin, X.; Hoffman, A.S.; Stayton, P.S. Poly(N-isopropylacrylamide-co-propylacrylic acid) copolymers that respond sharply to temperature and $\mathrm{pH}$. Biomacromolecules 2006, 7, 1381-1385. [CrossRef] [PubMed]

16. Kim, J.; Nayak, S.; Lyon, L.A. Bioresponsive hydrogel microlenses. J. Am. Chem. Soc. 2005, 127, $9588-9592$. [CrossRef] [PubMed]

17. Kim, J.; Serpe, M.J.; Lyon, L.A. Hydrogel microparticles as dynamically tunable microlenses. J. Am. Chem. Soc. 2004, 126, 9512-9513. [CrossRef] [PubMed]

18. Kim, J.; Park, Y.; Brown, A.C.; Lyon, L.A. Direct observation of ligand-induced receptor dimerization with a bioresponsive hydrogel. RSC Adv. 2014, 4, 65173-65175. [CrossRef]

19. Serpe, M.J.; Yarmey, K.A.; Nolan, C.M.; Lyon, L.A. Doxorubicin uptake and release from microgel thin films. Biomacromolecules 2005, 6, 408-413. [CrossRef] [PubMed]

20. Nolan, C.M.; Serpe, M.J.; Lyon, L.A. Thermally modulated insulin release from microgel thin films. Biomacromolecules 2004, 5, 1940-1946. [CrossRef] [PubMed]

21. Kikuchi, A.; Okano, T. Pulsatile drug release control using hydrogels. Adv. Drug Deliv. Rev. 2002, 54, 53-77. [CrossRef]

22. Kim, Y.M.; Song, S.C. Targetable micelleplex hydrogel for long-term, effective, and systemic sirna delivery. Biomaterials 2014, 35, 7970-7977. [CrossRef] [PubMed]

23. Suh, K.Y.; Langer, R.; Lahann, J. A novel photoderinable reactive polymer coating and its use for microfabrication of hydrogel elements. Adv. Mater. 2004, 16, 1401-1405. [CrossRef]

24. Kim, J.; Serpe, M.J.; Lyon, L.A. Photoswitchable microlens arrays. Angew. Chem. Int. Ed. 2005, 44, $1333-1336$. [CrossRef] [PubMed]

25. Shimoboji, T.; Larenas, E.; Fowler, T.; Kulkarni, S.; Hoffman, A.S.; Stayton, P.S. Photoresponsive polymer-enzyme switches. Proc. Natl. Acad. Sci. USA 2002, 99, 16592-16596. [CrossRef] [PubMed]

26. Mende, M.; Petzold, G.; Buchhammer, H.M. Polyelectrolyte complex formation between poly(diallyldimethylammonium chloride) and copolymers of acrylamide and sodium- acrylate. Colloid Polym. Sci. 2002, 280, 342-351. [CrossRef]

27. Papadakis, C.; Tsitsilianis, C. Responsive hydrogels from associative block copolymers: Physical gelling through polyion complexation. Gels 2017, 3, 3. [CrossRef] 
28. Antipov, A.A.; Sukhorukov, G.B.; Donath, E.; Mohwald, H. Sustained release properties of polyelectrolyte multilayer capsules. J. Phys. Chem. B 2001, 105, 2281-2284. [CrossRef]

29. Hoffman, A.S. Hydrogels for biomedical applications. Adv. Drug Deliv. Rev. 2002, 54, 3-12. [CrossRef]

30. Kamiya, N.; Klibanov, A.M. Controling the rate of protein release from polyelectrolyte complexes. Biotechnol. Bioeng. 2003, 82, 590-594. [CrossRef] [PubMed]

31. Decher, G.; Hong, J.D. Buildup of ultrathin multilayer films by a self-assembly process: III. Consecutive adsorption of anionic and cationic bipolar amphiphiles and polyelectrolytes on charged surfaces. Berichte der Bunsengesellschaft für Physikalische Chemie 1991, 95, 1430-1434. [CrossRef]

32. Decher, G. Fuzzy nanoassemblies: Toward layered polymeric multicomposites. Science 1997, 277, $1232-1237$. [CrossRef]

33. Gittins, D.I.; Caruso, F. Multilayered polymer nanocapsules derived from gold nanoparticle templates. Adv. Mater. 2000, 12, 1947-1949. [CrossRef]

34. Kato, N.; Schuetz, P.; Fery, A.; Caruso, F. Thin multilayer films of weak polyelectrolytes on colloid particles. Macromolecules 2002, 35, 9780-9787. [CrossRef]

35. Park, M.K.; Xia, C.; Advincula, R.C.; Schuetz, P.; Caruso, F. Cross-linked, luminescent spherical colloidal and hollow-shell particles. Langmuir 2001, 17, 7670-7674. [CrossRef]

36. Priftis, D.; Xia, X.; Margossian, K.O.; Perry, S.L.; Leon, L.; Qin, J.; de Pablo, J.J.; Tirrell, M. Ternary, tunable polyelectrolyte complex fluids driven by complex coacervation. Macromolecules 2014, 47, 3076-3085. [CrossRef]

37. Priftis, D.; Farina, R.; Tirrell, M. Interfacial energy of polypeptide complex coacervates measured via capillary adhesion. Langmuir 2012, 28, 8721-8729. [CrossRef] [PubMed]

38. Spruijt, E.; Sprakel, J.; Cohen Stuart, M.A.; van der Gucht, J. Interfacial tension between a complex coacervate phase and its coexisting aqueous phase. Soft Matter 2010, 6, 172-178. [CrossRef]

39. Spruijt, E.; Sprakel, J.; Lemmers, M.; Stuart, M.A.C.; van der Gucht, J. Relaxation dynamics at different time scales in electrostatic complexes: Time-salt superposition. Phys. Rev. Lett. 2010, 105, 208301. [CrossRef] [PubMed]

40. Cho, J.; Caruso, F. Polymeric multilayer films comprising deconstructible hydrogen-bonded stacks confined between electrostatically assembled layers. Macromolecules 2003, 36, 2845-2851. [CrossRef]

41. Chung, A.J.; Rubner, M.F. Methods of loading and releasing low molecular weight cationic molecules in weak polyelectrolyte multilayer films. Langmuir 2002, 18, 1176-1183. [CrossRef]

42. Chluba, J.; Voegel, J.C.; Decher, G.; Erbacher, P.; Schaaf, P.; Ogier, J. Peptide hormone covalently bound to polyelectrolytes and embedded into multilayer architectures conserving full biological activity. Biomacromolecules 2001, 2, 800-805. [CrossRef] [PubMed]

43. Lvov, Y.; Decher, G.; Sukhorukov, G. Assembly of thin-films by means of successive deposition of alternate layers of dna and poly(allylamine). Macromolecules 1993, 26, 5396-5399. [CrossRef]

44. Lvov, Y.; Haas, H.; Decher, G.; Mohwald, H.; Mikhailov, A.; Mtchedlishvily, B.; Morgunova, E.; Vainshtein, B. Successive deposition of alternate layers of polyelectrolytes and a charged virus. Langmuir 1994, 10, 4232-4236. [CrossRef]

45. Yoo, M.K.; Sung, Y.K.; Cho, C.S.; Lee, Y.M. Effect of polymer complex formation on the cloud-point of poly(isopropyl acrylamide) (PNIPAAm) in the poly(nipaam-co-acrylic acid): Polyelectrolyte complex between poly(acrylic acid) and poly(allylamine). Polymer 1997, 38, 2759-2765.

46. Yoo, M.K.; Sung, Y.K.; Lee, Y.M.; Cho, C.S. Effect of polyelectrolyte on the lower critical solution temperature of poly( $N$-isopropyl acrylamide) in the poly(nipaam-co-acrylic acid) hydrogel. Polymer 2000, 41, 5713-5719. [CrossRef]

47. Reineke, T.M.; Davis, M.E. Structural Effects of Carbohydrate-Containing Polycations on Gene Delivery. 1. Carbohydrate Size and Its Distance from Charge Centers. Bioconj. Chem. 2003, 14, 247-254. [CrossRef] [PubMed]

48. Rungsardthong, U.; Ehtezazi, T.; Bailey, L.; Armes, S.P.; Garnett, M.C.; Stolnik, S. Effect of polymer ionization on the interaction with DNA in nonviral gene delivery systems. Biomacromolecules 2003, 4, 683-690. [CrossRef] [PubMed]

49. Rao, G.V.R.; Konishi, T.; Ise, N. Ordering in poly(allylamine hydrochloride) gels. Macromolecules 1999, 32, 7582-7586. [CrossRef] 
50. Gan, D.; Lyon, L.A. Tunable swelling kinetics in core-shell hydrogel nanoparticles. J. Am. Chem. Soc. 2001, 123, 7511-7517. [CrossRef] [PubMed]

51. Gan, D.; Lyon, L.A. Interfacial nonradiative energy transfer in responsive core-shell hydrogel nanoparticles. J. Am. Chem. Soc. 2001, 123, 8203-8209. [CrossRef] [PubMed]

52. Jones, C.D.; Lyon, L.A. Synthesis and characterization of multiresponsive core-shell microgels. Macromolecules 2000, 33, 8301-8306. [CrossRef]

53. Schaefer, D.W.; Han, C.C. Dynamic Light Scattering, 1st ed.; Pecora, R., Ed.; Springer: New York, NY, USA, 1985; pp. 347-362.

54. Evans, D.F.; Wennerstrom, H. The Colloidal Domain: Where Physics, Chemistry, Biology, and Technology Meet, 2nd ed.; VCH Publishers, Inc.: New York, NY, USA, 1998; pp. 187-236.

55. Elimelech, M.; Williams, R.A.; Jia, X.; Gregory, J. Particle Deposition and Aggregation: Measurement, Modeling and Simulation, 1st ed.; Butterworth-Heinemann Ltd.: Oxford, UK, 1997; p. 448.

56. Sato, T.; Ruch, R. Stabilization of Colloidal Dispersions by Polymer Adsorption, 1st ed.; Marcel Dekker Inc.: New York, NY, USA, 1980; Volume 9, p. 155.

57. Gregory, J. Rates of flocculation of latex particles by cationic polymers. J. Colloid Interface Sci. 1973, 42, 448-456. [CrossRef]

58. Saunders, B.R.; Vincent, B. Microgel particles as model colloids: Theory, properties and applications. Adv. Colloid Interface Sci. 1999, 80, 1-25. [CrossRef]

(C) 2017 by the authors; licensee MDPI, Basel, Switzerland. This article is an open access article distributed under the terms and conditions of the Creative Commons Attribution (CC BY) license (http:/ / creativecommons.org/licenses/by/4.0/). 